
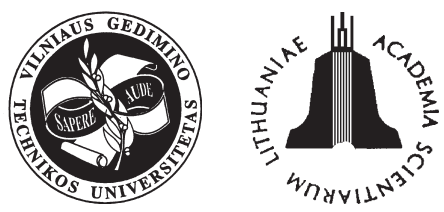

ISSN 1648-4142 TRANSPORT

www.transport.vtu.lt

\title{
INVESTIGATION OF HYDRODYNAMIC PROCESSES OF THE EXTINGUISHING DEVICE
}

\author{
Marijonas Bogdevicius ${ }^{1}$, Vladimiras Suslavicius ${ }^{2}$ \\ Dept of Transport Technological Eguipment, Vilnius Gediminas Technical University, Plytinès g. 27, \\ LT-10105VVilnius-16, Lithuania.Tel. (+370 5)2744782; e-mail:1'marius@ti.vtu.lt; ${ }^{2} v$.suslavicius@vpgt.lt
}

Received 12 April 2005; accepted 30 September 2005

\begin{abstract}
The main aim of the investigation is to develop an approach to investigate hydrodynamic processes in the extinguishing device. The mathematical model of the extinguishing device is presented where the flow of fluid and gas and the interaction of liquid with gas are taken into account. The flow of fluid in a hydraulic system is described by a system of equations of a hyperbolic type which is solved by a characteristics method. An instance of the mathematical simulation of the activity extinguishing device is shown. The dependence of recoil force is obtained.
\end{abstract}

Keywords: extinguishing device, gas, liquid, dynamics, recoil force, numerical methods.

\section{Introduction}

Extinguishing systems comprise systems designed for the supply of extinguishing materials (extinguishants) to fight fires. Water is the most available and the most frequently used extinguishing material since times remembered. Water is distinguished for its distinctive physical and chemical qualities. For instance, it is noted for its heat absorption characteristics that the majority of natural substances lack. For many years people have been trying to find better ways of delivering water to the scene of an accident and to use it in the most effective way in fire fighting. It is not infrequent that damages resulting from inefficient application of water exceed those done by fire to the burned down property and other valuables. Water used in fire fighting tends to leak out and pollute the environment and severely deteriorate the ecological conditions in general. Although various up-to-date pumps, hoses, nozzles and sprayers are used to extinguish fires, water-based fire extinguishing technologies have not reached the top level of performance. Even using modern centrifugal pumps it is not possible to prevent water spillage on the scene of a fire accident. In fact, this leaking water is not involved in fire extinction, but is being contaminated and wasted. This is due to the fact that part of this water fails to absorb the entire possible heat and tends to evaporate. This is also explained by the high tension of water surface which does not allow it to penetrate into the burning substances. It is evident that the more we atomize the water, the more of the surface area we will be able to obtain from the same volume of water which will directly contact with the fire heat and thus water properties will be used more efficiently. For instance, if water were poured as if from the bucket, its features would be used only at $5 \%$ efficiency. Thus, the increase of the surface area of the extinguishing water augments the efficiency of the water consumption as well. A simple way to increase the extinguishing water surface area is to atomize water into fine drops. The smaller the drops are developed, the better use of the water properties can be implemented and less water is consumed in fire fighting. The pressure energy of the pressurized and out-flowing water through the opening (i.e. fire nozzle) is transformed into jet kinetic energy. If we use the energy of the compressed air or other gases to eject water through the nozzle (instead of the compressed water energy) the jet speeds will be much faster. The water droplet speed within the jet sprayed out in an ordinary way reaches tens $\mathrm{m} / \mathrm{s}$, while using the compressed air energy the water droplet speed can reach hundreds $\mathrm{m} / \mathrm{s}$. Furthermore, because of such speeds, water spray is atomized into fine droplets due to air resistance (even up to 2 microns in diameter). Consequently, the extinguishing water covered area enlarges as well as the water efficiency. The devices with such properties can be usable in portable version. That is very important for extinguishing small fires. Small fires by statistics reach more than $50 \%$ of all fires. When water is sup- 
plied in fine droplets it is possible to reach the use of all of its properties as close as $100 \%$. In addition, the factor of the possible damage of the property and other valuables by water flooding is eliminated completely: facilities that are not within the extinguishing area remain safe from being flooded. The majority of fires could be addressed while using portable effective extinguishing devices.

The extinguishing device based on the use of the compressed air energy for ejecting extinguishing water could be expressed as follows (Fig 1):

- Compressed air compartment is filled up from the air container;

- Water compartment is filled up from the water tank;

- When the fast reaction valve is opened, compressed air and water compartments get merged;

- Water being under air pressure is ejected within a very short time (from several to several tens of mili-seconds) to the focus of fire;

- Further on the process is repeated from the beginning.

Studies of such extinguishing technologies have not been completed yet and need to be further updated and tested. The main parts of the investigation are:

- Process of extinguishing (water) media delivery to fire;

Recoil of the extinguishing device during operation.

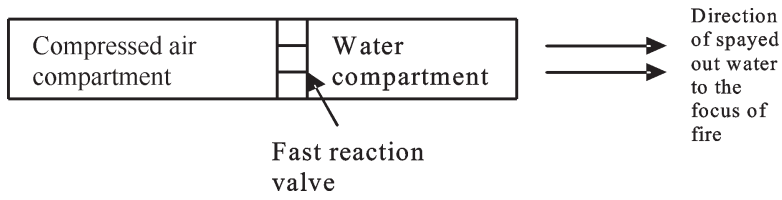

Fig 1. Principle scheme of the extinguishing device based on the use of compressed air energy

\section{Mathematical model of the extinguishing device}

The extinguishing device consists of two chambers (air container and water compartment) and two valves. The first valve is a fast reaction valve. The second valve opens when pressure reaches particular pressure. When the fast reaction valve begins to open the second chamber divides into two volumes. In the first volume there is high pressure of air and in the second volume there is high pressure of water.

A dynamic model of the extinguishing device is shown in Fig 2. In the air container the pressure is $p_{1}(t)$ and the volume of air container is $V_{1}$.

Cross-section area $S v_{1}$ of the first valve is function of time (Fig 3). Cross-section area $\mathrm{Sv}_{2}$ of the second valve depends on pressure $p(t, x=L)$ (Fig $4)$. In the second air volume $V_{2}(t)$ the pressure is $p_{2}(t)$. The second air volume and water compartment

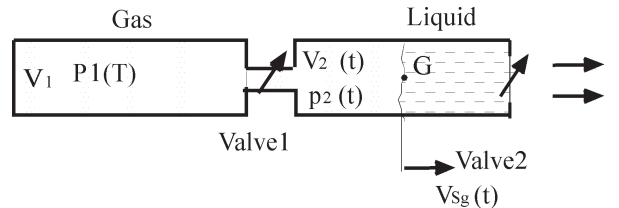

Fig 2. Diagram of the extinguishing device

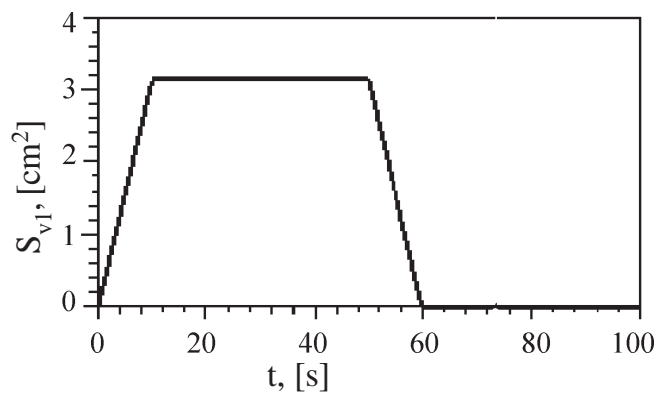

Fig 3. Cross-section area of the first valve

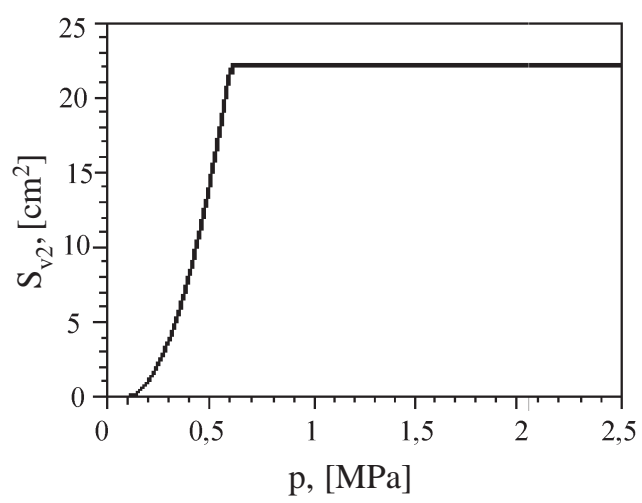

Fig 4. Cross-section area of the second valve

are separated by surface G (see Fig 2). According to the first law of thermodynamics the whole thermal energy moved with gas is spent for the change of internal energy and for the work of the expansion of gas in volume.

The change of pressure of constant volume $\left(V_{1}=\right.$ const $)$ of air container is determined from the following equation:

$$
\frac{d p_{1}}{d t}=-\frac{\gamma R T_{1}}{V_{1}} G_{12},
$$

where $G_{12}$ is mass charge of gas (air), determined by the formula Sen-Venan and Vencel [1]:

$$
\begin{aligned}
& G_{12}=\mu_{1} S_{v 1} p_{1} K_{1}\left(T_{1}\right) \varphi\left(\sigma=\frac{p_{2}}{p_{1}}\right) ; \\
& K_{1}(T)=\sqrt{\frac{2 \gamma}{(\gamma-1) R T_{1}}} ;
\end{aligned}
$$




$$
\varphi(\sigma)=\sqrt{\sigma^{\frac{2}{\gamma}}-\sigma^{\frac{\gamma+1}{\gamma}}},
$$

$S_{v 1}$ is cross-section area of the first valve; $\mu_{1}$ is factor of the charge; $\gamma$ - ration of specific heat; $R$ - gas constant; $T$ - temperature.

The change of pressure of the second volume is determined from the following equation:

$$
\frac{d p_{2}}{d t}=\frac{\gamma R T_{1}}{V_{2}(t)} G_{12}-\frac{\gamma p_{2}}{V_{2}(t)} \frac{d V_{2}}{d t},
$$

where $V_{2}(t)=V_{20}+S x_{G} ; V_{20}$ is initial volume; $S$ is cross-section area; $x_{G}$ is coordinate of point $\mathrm{G}$ (see Fig 2).

The liquid movement is considered as one-dimensional, i.e. all local velocity is equal to average velocity and unsettled. Velocity and pressure depend on longitude coordinate and time. Such liquid movement is characterized by the wave of increased and reduced pressure which spreads from the place of change in each pressure vibration cross-section and in deformation of pipeline walls.

The movement and continuity equations of viscous, compressible fluid in a pressure pipe have the following form [1-3]:

$$
\begin{aligned}
& \frac{\partial}{\partial t}[S(x) \rho]+\frac{\partial}{\partial x}[S(x) \rho v]=F_{1}(x), \\
& \frac{\partial}{\partial t}[S(x) \rho v]+\frac{\partial}{\partial x}\left[S(x)\left(p+\rho v^{2}\right)\right]=F_{2}(p, v),
\end{aligned}
$$

where $\rho$ is density of liquid.

An equation of one-dimensional movement of fluid can be written as the system quasi-linear differential equations:

$$
[A]\left\{\frac{\partial u}{\partial t}\right\}+[B]\left\{\frac{\partial u}{\partial x}\right\}=\{f\},
$$

where

$$
[A]=\left[\begin{array}{cc}
1 & 0 \\
0 & 1
\end{array}\right] ; \quad[B]=\left[\begin{array}{cc}
v & a^{2} \rho \\
\frac{1}{\rho} & v
\end{array}\right],\{u\}^{T}=[p, v],
$$

$v, p$-speed and fluid pressure; $a$ is sound velocity in the liquid with a certain amount of gas, which is stored in the elastic pipeline, is equal to:

$$
a=\sqrt{\frac{K(p) / \rho}{1+\frac{K(p) \cdot d}{E \cdot e}+\frac{\varepsilon}{\gamma}\left[\frac{K(p)}{\gamma p}-1\right]}}
$$

where $K(p)$-bulk modulus of elasticity of liquid, $\rho$ density of liquid, $E$ - modulus of elasticity of a pipeline, $d$-internal diameter of a pipeline, $e-$ thickness of a wall of a pipeline, $\gamma$-index of adiabatic process, $\varepsilon$ - ratio of gas volume in the liquid and the total volume of liquid (mixture).

Differential equations of liquid movement in the cylinder are solved by a characteristic method [1,2]. The main idea of the characteristic method is the fact that unknown variable speed and liquid pressure at instant moment of time $t+\Delta t$ are determined according to these parameters at the moment of time (Fig 5).

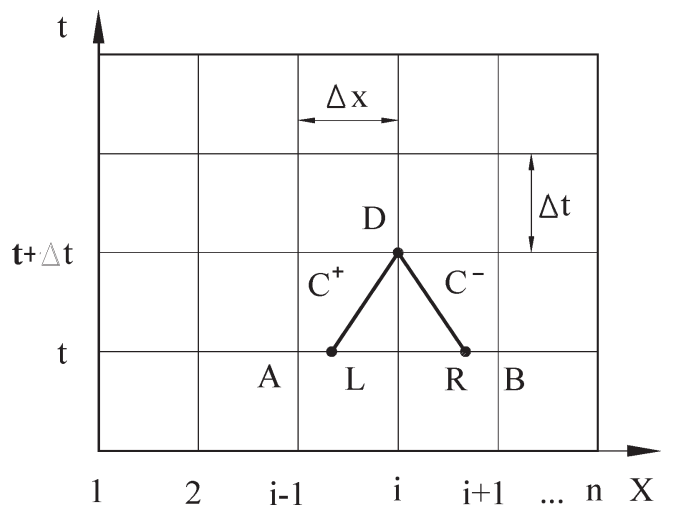

Fig 5. Circuit of liquid parameters determination of point $D$

Equating the determinant of matrix (13) to zero, we shall receive the equation:

$$
\left|[B]-[A] \frac{d x}{d t}\right|=0
$$

which allows to determine $\frac{d x}{d t}$ derivative which determines characteristic direction. If this equation has $n$ various real roots $d x / d t=\lambda_{i}(i=1,2)$, the initial system of the differential equations is referred to as hyperbolic. The inclination tangent $\lambda_{i}$ to the characteristic depends not only on coordinates, but also on solution $\{u\}$.

Inserting expressions $[A]$ and $[B]$ from matrices (7) into equation (9) and having solved it, we receive three equations of characteristics

$$
C^{+}: \frac{d x}{d t}=v+a ; C^{-}: \frac{d x}{d t}=v-a .
$$

Compatibility conditions of characteristics are equal to [4-6]:

$$
\begin{aligned}
& C^{+}: \frac{d v}{d t}+\frac{1}{a \rho} \frac{d p}{d t}=\frac{f_{1}}{a \rho}+f_{2}, \\
& C^{-}: \frac{d v}{d t}-\frac{1}{a \rho} \frac{d p}{d t}=-\frac{f_{1}}{a \rho}+f_{2},
\end{aligned}
$$




$$
\{f\}=\left\{\begin{array}{c}
0 \\
-\frac{\tau \Pi(x)}{S(x) \rho}-a_{x}
\end{array}\right\},
$$

where $\tau$ - shear stresses on the inner surface of pipeline; $a_{x}$-acceleration along $\mathrm{x}$ axis; $S(x)$ and $\Pi(x)$ are cross-section area and perimeter of pipeline.

Pressure and velocity at point $D$ at the moment of time are determined from nonlinear algebraic equation system

$$
\begin{aligned}
& C^{+}: \Phi_{1}=v_{D}-v_{L}+\frac{1}{2}\left(p_{D}-p_{L}\right)\left[r_{1 L}+r_{1 D}\right]- \\
& \frac{\Delta t}{2}\left[r_{2 L}+r_{2 D}\right]-\frac{\Delta t}{2}\left[r_{3 L}+r_{3 D}\right]=0, \\
& C^{-}: \Phi_{2}=v_{D}-v_{R}-\frac{1}{2}\left(p_{D}-p_{R}\right)\left[r_{1 R}+r_{1 D}\right]+ \\
& \frac{\Delta t}{2}\left[r_{2 R}+r_{2 D}\right]-\frac{\Delta t}{2}\left[r_{3 R}+r_{3 D}\right]=0,
\end{aligned}
$$

where $r_{1}=\frac{1}{\rho a} ; \quad r_{2}=-a^{2} \rho v \frac{d S}{d x} / S ;$

$$
\begin{gathered}
r_{3}=-a_{x}-\frac{\Pi \lambda(R e) v|v|}{8 S} ; \\
\lambda(\operatorname{Re})=\left\{\begin{array}{l}
\frac{75}{R e}, \text { when } \quad R e \leq 2320 ; \\
\frac{0,31464}{R e^{0,25}}, \text { when } \quad R e>2320,
\end{array}\right.
\end{gathered}
$$

$\lambda(R e)$ is coefficient of pressure losses along a pipe.

The system of equations (2) and (3) is solved by Newton method:

$$
[J]_{i}\{\Delta Y\}_{i}=-\{\Phi(Y)\}_{i},
$$

where $\{Y\}^{T}=\left[p_{D}, v_{D}\right] ;\{\Phi\}^{T}=\left[\Phi_{1}, \Phi_{2}\right]$.

The potential energy of the gas in a high-pressure volume is transformed to kinetic energy of the liquid. For accuracy the simulation of the interaction of the gas with liquid in the case of interaction is considered (Fig 6). At point $G$ pressure $p_{S G}$ and velocity $v_{S G}$ are determined from the system of equations (3) and (15). The x coordinate of point $G$ is determined from the following expression:

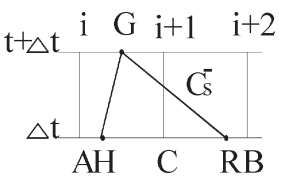

Fig 6. Circuit of liquid parameters determination at point $G$

$$
x_{G}(t+\Delta t)=x_{H}(t)+\Delta t v_{S G} .
$$

The diagram of forces acting on the extinguishing device when out-flowing water through the open-

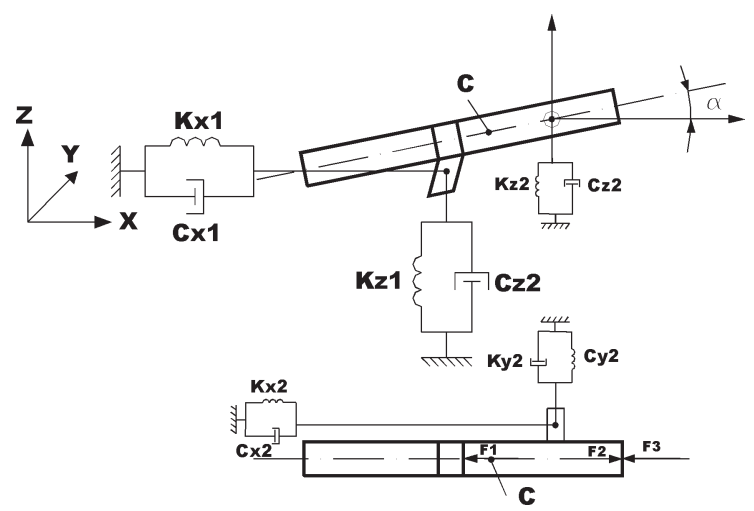

Fig 7. Diagram of forces acting on the extinguishing device

ing (i.e. fire nozzle) valve is shown in Fig 7.

The system of equation describing the extinguishing device is as follows:

$$
[M]\{\ddot{q}\}+[C]\{\dot{q}\}+[K]\{q\}=\{F(t, p, v)\},
$$

where $[M],[C],[K]$ are matrices of mass, damping, stiffness, respectively; $\{q\},\{\dot{q}\},\{\ddot{q}\}$ are vectors of displacement, velocity and acceleration, respectively; $\{F(t, p, v)\}$ is vector of forces and moments.

\section{Numerical results}

An example of the extinguishing device is considered. The following data of the extinguishing device were used: the length of water compartment is $0,420 \mathrm{~m}$, the volume of air container is equal to $1,5 \cdot 10^{-3} \mathrm{~m}^{3}$, initial pressure in the air container is 2,50 $\mathrm{MPa}$, inner diameter of water compartment is equal to $0,060 \mathrm{~m}$. Time integration step is equal to $2,0 \cdot 10^{-6} \mathrm{~s}$. The length of water compartment is divided in to 84 elements [7].

The simulation results of hydrodynamic parameters are given in Fig 8.

The forces acting on the extinguishing device are shown in Fig 9 and Fig 10.

The displacement and velocity of the extinguishing device are shown in Fig 11 and Fig 12. 

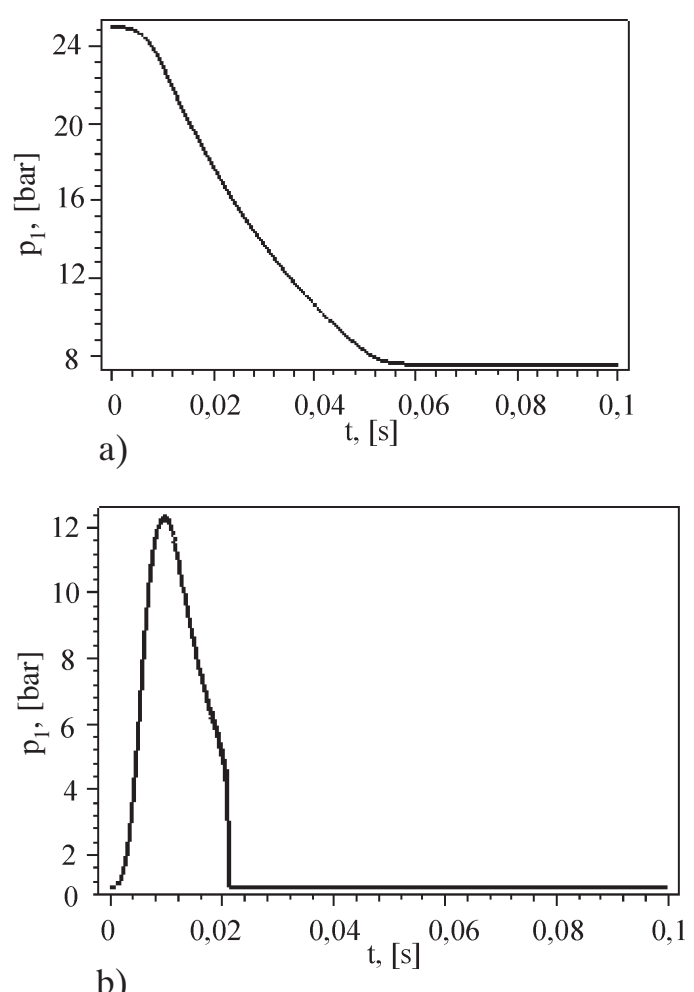

b)
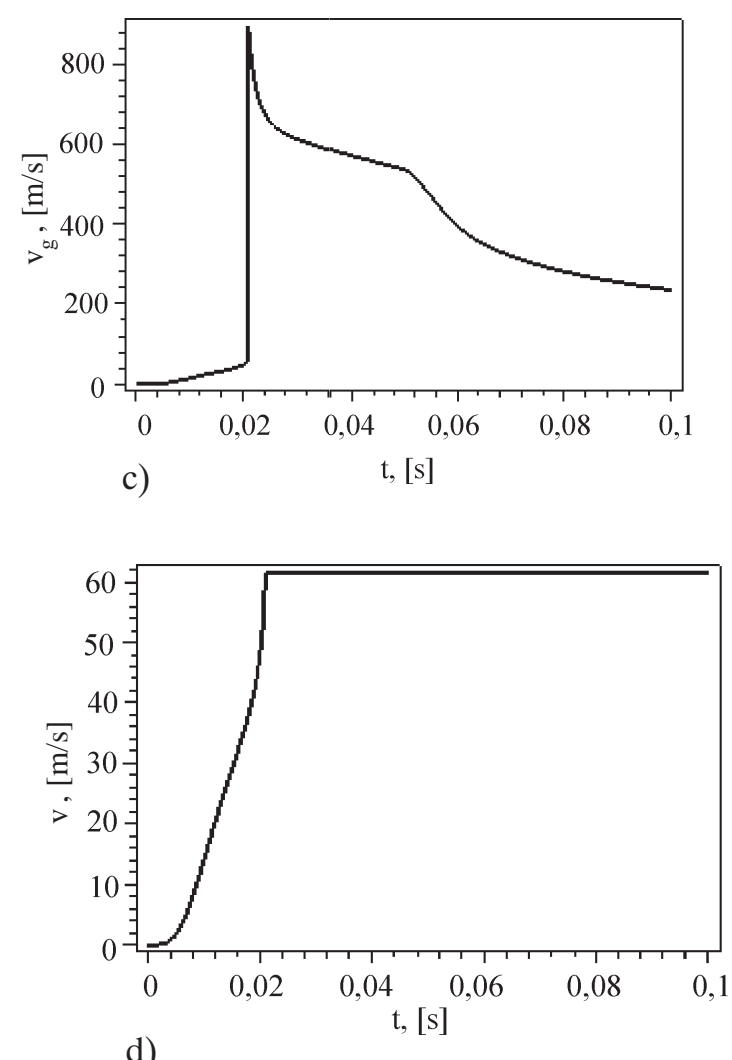

Fig 8. The parameters of the extinguishing device:

a - change of pressure in the air container;

$\mathrm{b}$ - change of air pressure in the second volume;

c - change of liquid velocity at the point $G$;

$\mathrm{d}$ - change of liquid velocity at the end of pipeline

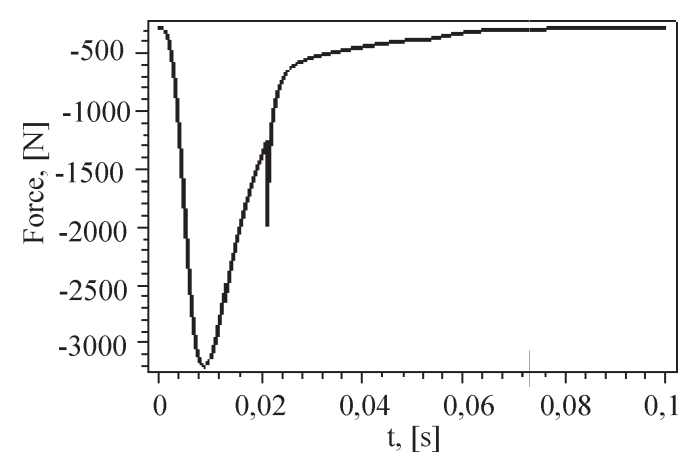

Fig 9. Dependence of pressure and jet-propolsion force upon time

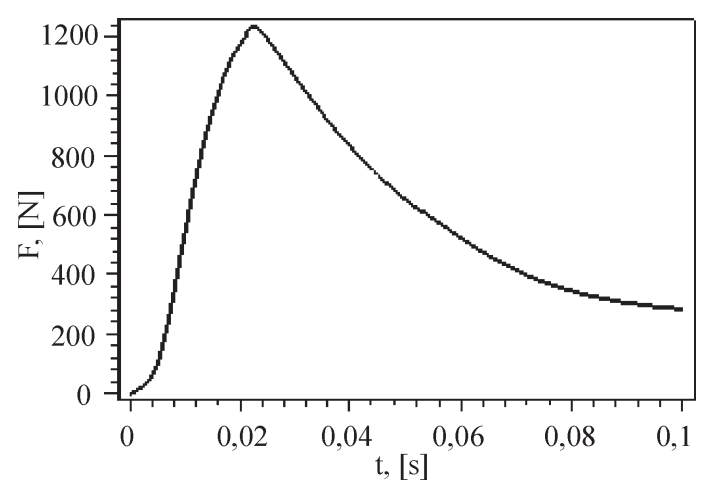

Fig10. Dependence of recoil force upon time

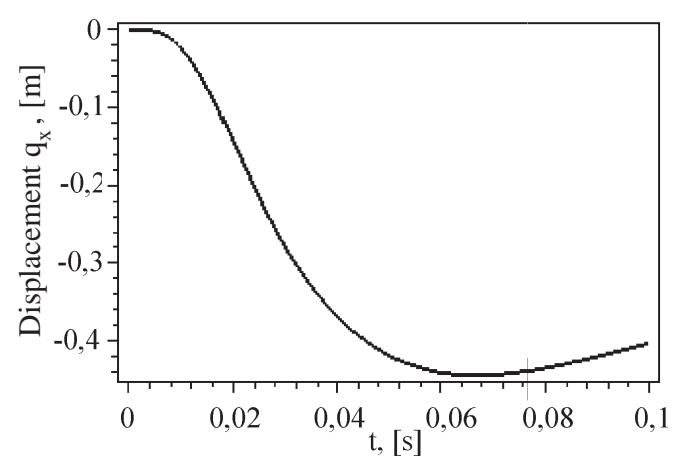

Fig 11. Displacement of extinguishing device

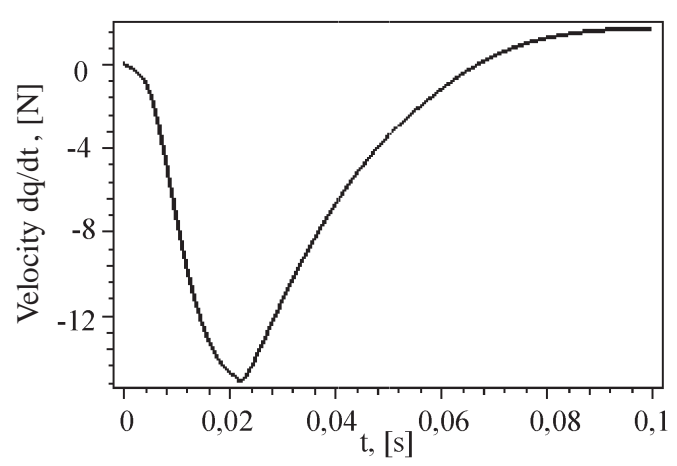

Fig 12. Velocity of the extinguishing device 


\section{Conclusions}

1. A new approach to simulating hydrodynamic processes of the extinguishing device has been developed. The composed mathematical model of the extinguishing device takes into account wave motion of a liquid.

2. Differential equations describing hydrodynamic processes inside the extinguishing device help analyze the movement of liquid and gas better and more precisely.

3. At the end of a pipeline of the extinguishing device the maximum velocity of the liquid when initial pressure is equal to $2.5 \mathrm{MPa}$ reaches $60 \mathrm{~m} / \mathrm{s}$.

\section{References}

1. Bogdevicius, M. Pump hydraulic system simulation by the characteristic method. Transportas (Transport Engineering). Vilnius: Technika, 1997, No 2(15), p. 30-37 (in Russian).

2. Bogdevicius, M. Calculation of a non-stationary move- ment of a liquid in elastic-plastic and elastic-viscousplastic pipelines. University Stuttgart, Institute of Hydraulic Machines, 1991. 48 p.

3. Bogdevicius, M. Simulation and Interaction of Mechanical and Hydraulic System. In: Proceedings of Tenth World Congress on the Theory of Mechines and Mechanisms. Oulu (Finland), 1999, p. 2110-2115.

4. Bogdevicius, M. Simulation of Dynamic Processes in Hydraulic, Pneumatic and Mechanical Drivers and their Elements. Vilnius: Technika, 2000. 96 p.

5. Aladjev, V.; Bogdevičius, M. Maple 6: Solution of the Mathematical, Statistical and Engineering - Physical Problems (Решение математических, статистических и инженерно-физических задач). Moscow: Laboratory of Basic Knowledges, 2001. 824 p. (in Russian).

6. Aladjev, V.; Bogdevicius, M.; Prentkovskis, O. New software for mathematical package Maple of releases 6, 7 and 8: Monograph. Vilnius: Technika, 2002. 404 p.

7. Bogdevičius, M.; Matuliauskas, A.; Mištinas, V. Spruogis, B.; Suslavičius, V. Dynamics of pneumatic and hydraulic transport technological equipment system. Transport, Vol XIX, No 1. Vilnius: Technika, 2004, p. 37-42. 